\title{
Fusion to chicken C3d enhances the immunogenicity of the M2 protein of avian influenza virus
}

\author{
Zhenhua Zhang ${ }^{1,2}$, Yongqing Li*+2, Shufang X ${ }^{2}$, Fuyong Chen ${ }^{*+1}$, Li Zhang², Beiyu Jiang² and Xiaoling Chen²
}

\begin{abstract}
Background: Current vaccines to avian influenzae virus (AIV), a highly contagious disease of birds, need to be constantly updated due to the high level of variation in the target antigens. Therefore, a vaccine that could induce broad cross protection against AIV is required. The M2 membrane protein is structurally conserved amongst AIV subtypes but tends in induce a poor immune response, whereas $C 3 \mathrm{~d}$ has been shown in many species to enhance immunogenicity. In this study, we investigated the potential of M2-avian C3d fusion proteins to provide effective immunity.
\end{abstract}

Results: We fused chicken complement C3d to SM2 (M2 protein with the transmembrane region deleted) of AIV and expressed four fusion proteins, GST (Glutathione S-transferase tagged proteins in pGEX expression vector) -C3d-sM2, GST-C3d-L2-sM2, GST-C3d-L1-C3d-sM2 and GST-C3d-L1-C3d-L2-sM2 were used to immunize mice. In addition, Specific pathogen free (SPF) chickens were inoculated with the plasmids pcDNA-sM2, pcDNA-C3d-L1-C3d-L2-sM2, GST-sM2 and GST-C3d-L1-C3d-L2-SM2. The immune response was monitored by an enzyme-linked immunosorbent assay (ELISA) for sM2 antibody, and all the test animals were challenged with A/chicken/Bei Jing/WD9/98 (H9N2) virus. Results revealed that the anti-sM2 antibody in mice and chickens vaccinated with these proteins was higher than the nonfused forms of SM2, the GST-C3d-L1-C3d-L2-SM2 groups have conferred the highest 30\% and 20\% protection ratio in mice and chickens respectively. In addition, the pCDNA-C3d-L1-C3d-L2-SM2 also enhances the antibody responses to $\mathrm{sM} 2$ compared to PCDNA-sM2 in chickens, and acquired $13.3 \%$ protection ratio.

Conclusion: These results indicated that chicken C3d enhanced the humoral immunity against AIV M2 protein either fused proteins expressed by the prokaryotic system or with the DNA vaccine. Nevertheless, in view of the poor protection ratio for these animals, we speculated that this is not a worthy developing of vaccine in these constructs.

\section{Background}

Complement is a protein system in the plasma of humans and animals [1]. After being activated, a series of important biological reactions generate several complement proteins that nonspecifically defend against invading pathogens [2]. While complement protein C3 is a central component of the innate immune system, it also plays an important role in stimulating the humoral immune response $[1,3]$. At the point of convergence of three dis-

* Correspondence: li.yongqing@bbsrc.ac.uk, vetchen@cau.edu.cn

2 Institute of Animal Husbandry and Veterinary Medicine, Beijing Academy of Agricultural and Forestry Sciences, Beijing 100097, China

1 College of Animal Medicine, China Agricultural University, Beijing 100094, China

+ Contributed equally

Full list of author information is available at the end of the article tinct pathways of complement activation, C3 is cleaved into C3a and C3b by the C3 convertase [4]. Further proteolytic cleavage of $\mathrm{C} 3 \mathrm{~b}$ results in the formation of $\mathrm{C} 3 \mathrm{c}$ and C3dg. The C3dg product can be further degraded by a variety of cellular proteases into C3d, a protein which attaches covalently to the surface of pathogens and upregulates B-cell responses [4,5]. Previous studies have demonstrated that C3d could enhance antigen recognition and specific immunoglobulin synthesis by antigenspecific B cells, as the antigen is taken up and processed via cell receptor 2 (CR2) by both antigen-specific and non-specific B cells [6]. Subsequent investigations showed that three copies of murine C3d could dramatically enhance antibody responses to specific antigen, being 100 -fold more effective than incomplete Freund's 
adjuvant $[7,8]$. Ross reported that C3d could enhance antibody responses directed toward a specific antigen encoded by a DNA vaccine [9]. A DNA vaccine expressing a fusion of hemagglutinin (HA) from influenza virus or measles virus fused to three copies of the murine homologue of C3d (mC3d) achieved an early and efficient immune response in mice. Fusion to C3d has been shown to increase the immunogenicity of the capsular polysaccharide antigen of Streptococcus pneumoniae [10]. Using DNA vaccination, various forms of envelope (Env) proteins of the human immunodeficiency virus type 1 (HIV1) fused at the carboxyl terminus with $C 3 d$ of murine complement, generated high-titer, long-lasting, neutralizing antibodies in mice [11]. In addition, the human homologue of C3d (hC3d) also enhanced anti-Env antibodies in rabbits when it was fused to sgp120 [12]. Recently, Wang reported that the bovine homologue of C3d (boC3d) coupled to the E2 envelope protein of bovine viral diarrhea virus greatly enhanced immunogenicity in mice [13]. Liu also reported that chicken C3dP29 linked to the F gene of Newcastle disease virus (NDV) enhanced immunogenicity in chickens [14]. Logan GJ found C3d (3)-fusion markedly increase antibody responses to the AAV-encoded model antigen (hen egg lysozyme) with greater than 50-fold enhancement in responses [15]. Comparison of the human, mouse and bovine C3d sequences showed $84.1 \%$ amino acid homology between hC3d and mC3d and 80.5\% homology between $\mathrm{hC} 3 \mathrm{~d}$ and boC3d, they either showed the function of immune adjuvant in mammalian model. Information on the function of avian C3d is scarce. Importantly, there are structural differences in the mammalian and avian immune systems, particularly the role of the bursa as one of the central immune organs in avian species.

Avian influenza (AI), caused by avian influenza virus (AIV), is a highly contagious disease of birds. Current AI vaccines induce antibodies against $\mathrm{HA}$ and neuraminidase (NA), two major surface glycoproteins expressed on the virus particles. However, due to rapid antigenic variation of $\mathrm{HA}$ and NA, AI vaccine can not protect avian against the new avian influenza virus strains. A vaccine that is less sensitive to the antigenic evolution of the virus would be a major improvement. As a result, vaccines have to be updated continuously to prevent disease emerging due to new viral strains. Hence, a vaccine that could induce broad cross protection against AIV would be desirable.

The Matrix protein 2 (M2) is an integral tetrameric membrane protein of AIV. Natural M2 protein is present in a few copies in the virus particle but in abundance on virus-infected cells. In contrast to hemagglutinin and neuraminidase, M2 is almost nonimmunogenic, and its sequence is highly conserved in all diverse subtypes of AIV. Several investigations have shown that the M2 pro- tein has the potential to induce a broadly protective immunity against AIV. M2-specific antibodies have been shown to restrict virus growth in vitro and in vivo and thus have the potential of providing cross-reactive resistance to influenza type A virus infection [16]. Frace reported that vaccination with the protein M2 was found to raise M2-specific serum antibodies and enhance viral clearance in mice challenged with homologous and heterologous influenza A viruses [17]. Neirynck reported that the M2 domain was genetically fused to the hepatitis B virus core $(\mathrm{HBc})$ protein to create fusion gene coding for $\mathrm{M} 2 \mathrm{HBc}$, and intraperitoneal or intranasal administration of purified M2HBc particles to mice provided $90-100 \%$ protection against a lethal virus challenge [18]. Zhao designed a tetra-branched multiple antigenic peptide (MAP)-based vaccine, designated M2e-MAP, which contains the sequence overlapping the highly conserved extracellular domain of matrix protein 2 (M2e) of a HPAI H5N1 virus, animals test results showed that M2e-MAP vaccine induced strong M2e-specific IgG antibody responses following 3-dose immunization of mice with M2e-MAP in the presence of Freunds' or aluminium (alum) adjuvant [19]. Rao SS have shown that vaccination with M2 in recombinant DNA and/or adenovirus vectors or with adjuvants confers protection against lethal challenge in the absence of $\mathrm{HA}$, and also find that the protective efficacy of NP and M2 diminishes as the virulence and dose of the challenge virus are increased [20]. However, M2 is only a minor protein component of AIV, and tends to induce a poor immune response [21]. To overcome the shortcomings of M2-based vaccines, in this study we investigated the potential of M2-avian C3d fusion proteins to provide effective immunity.

\section{Results}

\section{Cloning and sequence analysis of the chicken C3d gene} fragment

Chicken C3d has not previously been cloned so it was necessary to clone and sequence the complement C3d fragment. A PCR product of approximately $1 \mathrm{~kb}$ was amplified from RNA extracted from chicken liver tissue. The fragment was cloned into the pMD18-T (Takara) plasmid and sequenced. Sequence analysis revealed that the amplified fragment was $993 \mathrm{bp}$ in length and that the chicken C3d gene was 897 bp in length. This nucleotide sequence for chicken C3d was submitted to the GenBank database (DQ291160). The chicken C3d sequence showed the following \% identities to the published sequences for C3d from: Arbor Acres chicken (EF632299) 99.8\%; human (NM_000064) 66.4\%; mouse (BC043338) 66.2\%; hamster (AB024425) 67.9\%; cow (AY630404) 67.2\%; rabbit (M32434) 67.0\%; pig (NM_214009) 66.8\%; chimpanzee (XM_512318) 66.4\% and sheep (AF038130) $66.9 \%$, as determined by Clustal W multiple alignment of 
nucleotide sequences. The predicted protein sequence of chicken C3d showed the following \% similarity to the published protein sequences for C3d from: Arbor Acres chicken, 100\%; human, 61.5\%; mouse, 61.9\%; hamster, 61.2\%; cow, 62.2\%; rabbit, 56.5\%; pig, 61.9\%; chimpanzee, $61.5 \%$ and sheep, $61.5 \%$, as determined by DNAStar ClustalW analysis. A phylogenetic tree of the C3d amino acid sequences is shown in Fig. 1.

\section{Construction and identification of the recombinant expression plasmids}

Recombinant expression plasmids pGEX-C3d-sM2, pGEX-C3d-L1-C3d-sM2, pGEX-C3d-L2-sM2, pGEXC3d-L1-C3d-L2-sM2 and pcDNA-sM2, pcDNA-C3d-L1C3d-L2-sM2 were constructed with gene expression cassettes, pGEX-5x-1 and pcDNA4.0-LacZ. EcoRI and XhoI restriction endonuclease digestion resulted in a linearized pGEX-5x-1plasmid (of about 4900 bp), expression cassettes DNA of two copies of cC3d and SM2 (of about 2100 bp) and gene cassettes DNA of one copy of cC3d and sM2 (of about $1200 \mathrm{bp}$ ).

\section{Expression and purification of fusion proteins}

E. coli BL21 (DE3) cells were transformed with the four prokaryotic expression plasmids detailed above. Cells were then induced by IPTG at $28^{\circ} \mathrm{C}$. The expression products were purified using the SKL method. Examination by $12 \%$ SDS-PAGE showed that the molecular weight of the single fused copy of cC3d was about $70 \mathrm{kDa}$, while the molecular weight of the two copies fused to cC3d was about $102 \mathrm{kDa}$, both of these sizes corresponding to the sizes expected (Fig. 2).

\section{SDS-PAGE and Western immunoblot detection of fusion proteins}

Western blot analysis was performed with purified GSTC3d-L1-C3d-L2-sM2, GST-C3d-L1-C3d-sM2, GST-C3d-

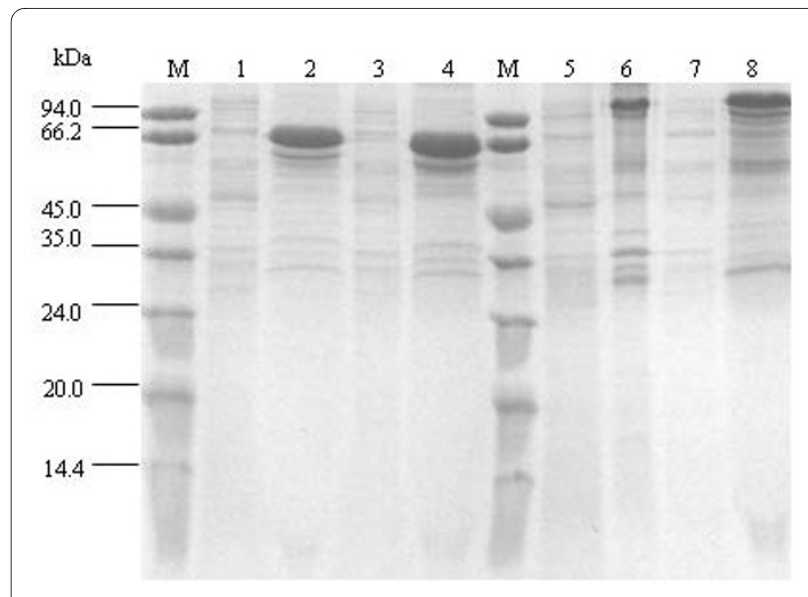

Figure 2 SDS-PAGE analysis of proteins expressed by the expression vectors containing one or two copies of $C 3 \mathrm{~d}$ fused to $\mathrm{SM} 2$ in E. coli, before and after induction. M: protein marker; lane 1: pGEXC3d-L2-sM2 before induction; lane 2: pGEX-C3d-L2-sM2 after induction; lane 3: pGEX-C3d-sM2 before induction; lane 4: pGEX-C3d-sM2 after induction; lane 5: pGEX-C3d-L1-C3d-sM2 before induction; lane 6: pGEX-C3d-L1-C3d-sM2 after induction; lane 7: pGEX-C3d-L1-C3d-L2SM2 before induction; lane 8: pGEX-C3d-L1-C3d-L2-sM2 after induction

L1-sM2 and GST-C3d-sM2 proteins and the anti-sM2 monoclonal antibody, which had been prepared in our laboratory. The band of interest was evident on Western blot analysis, indicating that the recombinant protein had maintained the antigenicity of the sM2 component (Fig. $3)$.

\section{Expression of recombinant eukaryotic plasmids in BHK-21 cells}

The constructed eukaryotic expression vectors pcDNAsM2, pcDNA-C3d-L1-C3d-L2-sM2 and pcDNA4.0 were transfected into BHK-21 cells. The indirect immunofluo-

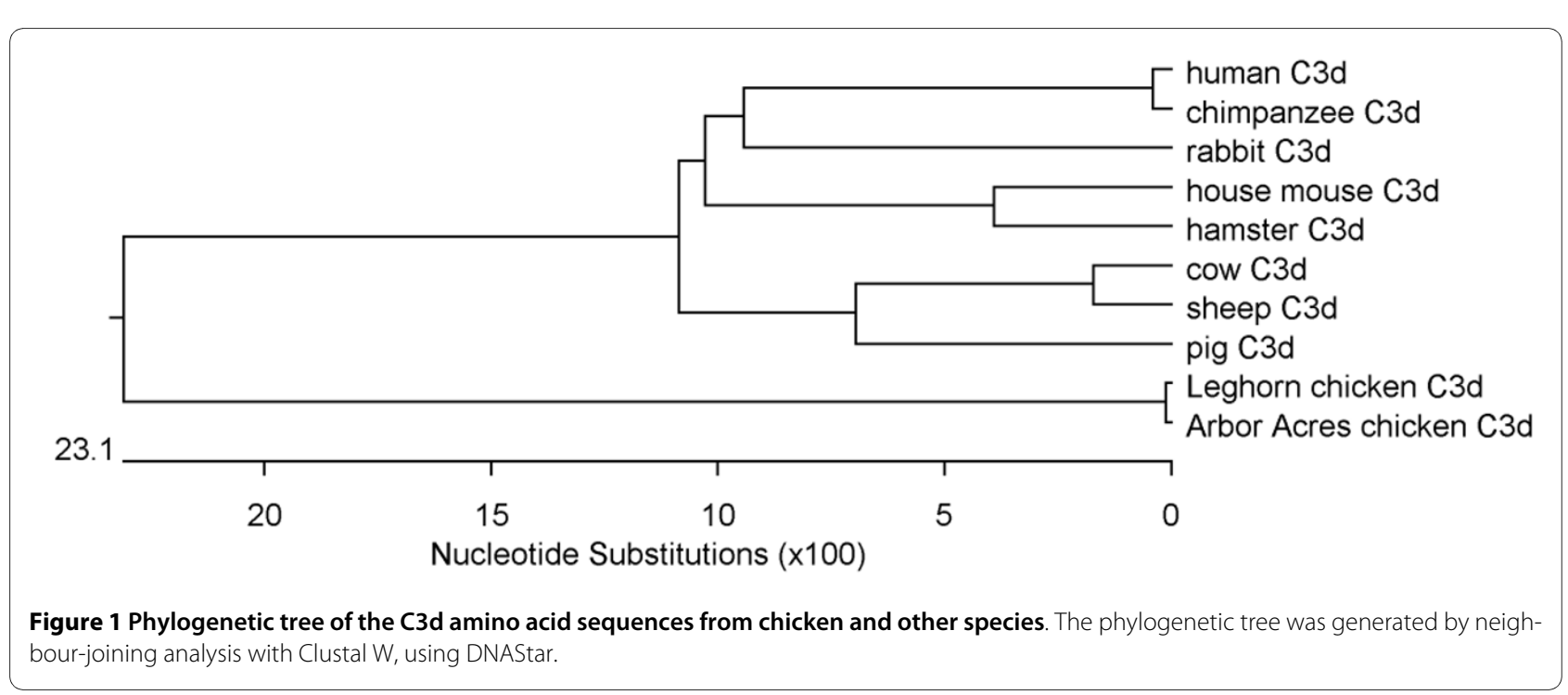




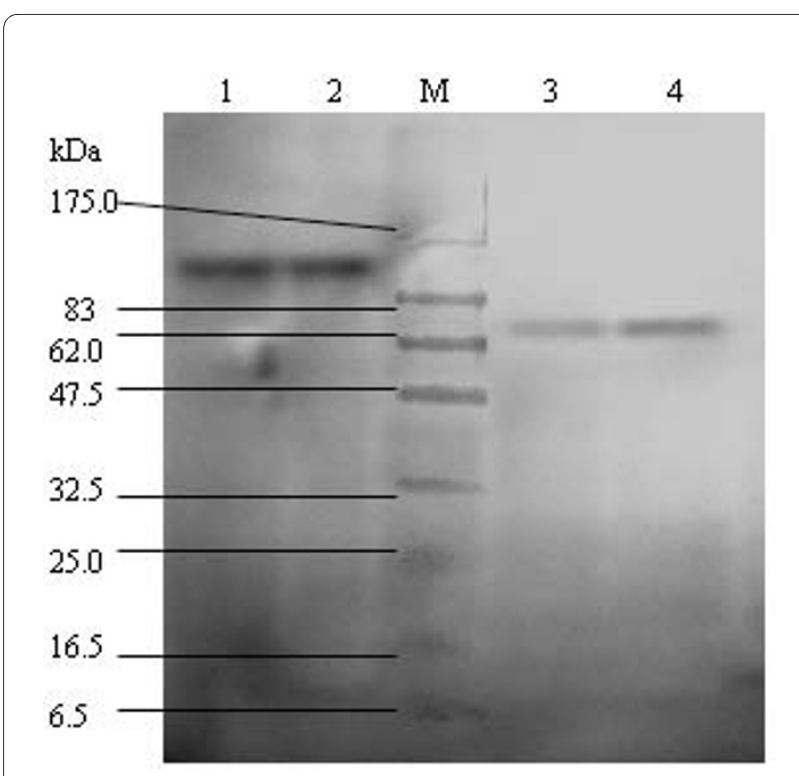

Figure 3 Western-blot analysis of the recombinant proteins GSTC3d-L1-C3d-L2-sM2, GST-C3d-L1-C3d-sM2, GST-C3d-L1-sM2 and GST-C3d-sM2. M: protein marker; lane 1: GST-C3d-L1-C3d-sM; lane 2: GST-C3d-L1-C3d-L2-sM2; lane 3: GST-C3d-sM2; lane 4: GST-C3d-L1sM2.

rescence test demonstrated that the sM2 protein was expressed in BHK-21 cells after the cells had been transfected with the pcDNA-sM2 and pcDNA-C3d-L1-C3dL2-sM2 eukaryotic expression plasmids (Fig. 4).

Chicken C3d increases the antibody titers of sM2 in BALB/C mice and protection against challenge

$\mathrm{BALB} / \mathrm{c}$ mice were immunized three times with purified GST-C3d-L1-C3d-L2-sM2, GST-C3d-L1-C3d-sM2, GST-C3d-L1-sM2, GST-C3d-sM2, GST-sM2 proteins and GST-sM2 mixed in IFA. The results showed that no anti-sM2 antibody was produced after the first and second immunizations with GST-C3d-L1-C3d-sM2, GSTC3d-L1-sM2 and GST-C3d-sM2 (Fig. 5). After the third

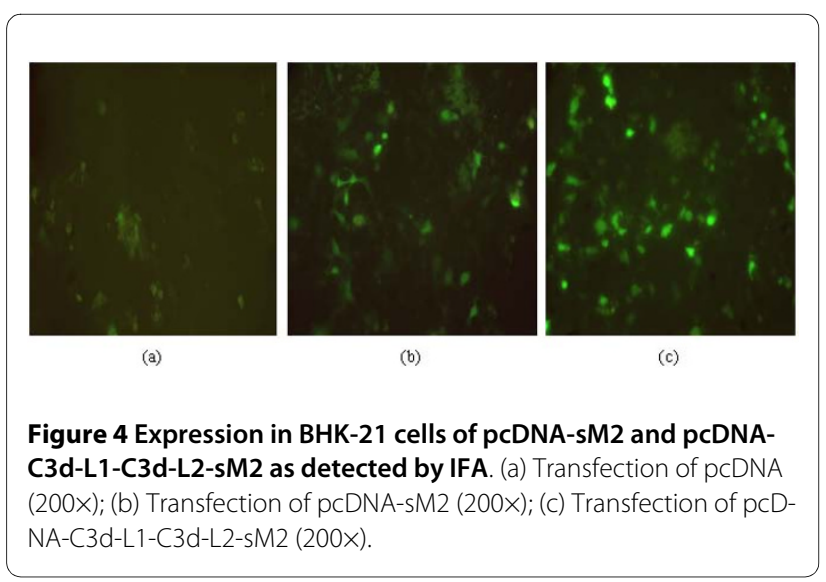

immunization, high level sM2 antibody titers appeared in six immunized groups. The difference between the GSTC3d-sM2, GST-C3d-L1-C3d-L2-sM2, GST-sM2 mixed in IFA immunized groups and the control group (which was GST-sM2) was very significant $(\mathrm{P}<0.01)$, the anti-sM2 antibody in mice primed with GST-C3d-L1-C3d-L2-sM2 was greater than that in GST-sM2 mixed in IFA group, but the difference between the two groups was not significant $(\mathrm{P}>0.05)$. The GST-sM2+IFA and GST-C3d-L1C3d-L2-sM2 groups have conferred the highest 30\% protection ratio in mice (Table 1 ), the GST-C3d-sM2 group provide $20 \%$ protection ratio secondly. Most of mice that attacked with H9N2 virus have showed some clinical signs after post-challenge, such as depressed, shiver and so on.

\section{Chicken C3d enhances the antibody titers of SM2 in SPF chickens and protection against challenge}

SPF chickens were immunized three times with GSTC3d-L1-C3d-L2-sM2, GST-sM2, GST-sM2 mixed in IFA and DNA vaccines of pcDNA-sM2 and pcDNA-C3d-L1C3d-L2-sM2. In the indirect ELISA, for the fusion protein groups, the difference between GST-C3d-L1-C3dL2-sM2, GST-sM2 mixed in IFA and GST-sM2 (control group) was very significant $(\mathrm{P}<0.01)$ after the second immunization. Indeed, the antibody titer of the GSTC3d-L1-C3d-L2-sM2 immunized group was higher than that of all the other groups at this point. The difference between GST-C3d-L1-C3d-L2-sM2 and GST-sM2 mixed in IFA group was not significant $(P>0.05)$ after the second or third immunization. After the third immunization, the difference between the experimental pcDNAC3d-L1-C3d-L2-sM2 group and the control pcDNA-sM2 group became significant $(\mathrm{P}<0.05)$ (Fig. 6). The GSTsM2+IFA group has the highest antibody titer and a $26.7 \%$ protection ratio (Table 2 ), the GST-C3d-L1-C3dL2-sM2 and pcDNA-C3d-L1-C3d-L2-sM2 groups provide $20 \%, 13.3 \%$ protection ratio respectively. No chicken that received $\mathrm{H} 9 \mathrm{~N} 2$ virus have showed obvious clinical signs on 5 days post challenge.

\section{Lymphocyte transformation assay (MTT method) of SPF chickens}

After the third immunization, peripheral blood lymphocytes were isolated from the SPF chickens and used in the lymphocyte transformation assay (see Table 3). Statistical analysis of the $\mathrm{OD}_{570}$ values showed that with respect to LPS-stimulated lymphocytes, the difference in OD values between the group immunized with GST-C3d1-L1-C3d2L2-sM2 fusion protein and control group immunized with GST-sM2 was very significant $(\mathrm{P}<0.01)$. The difference between the group immunized with pcDNA-C3dL1-C3d-L2-sM2 plasmid and the control group immunized with pcDNA-sM2 was also very significant $(\mathrm{P}<$ 
Table 1: Results of protection from immunized mice after challenge.

\begin{tabular}{llcc}
\hline Groups & Vaccine & Number of positive/total & Protection ratio (\%) \\
\hline 1 & GST-sM2 & $10 / 10$ & 0 \\
2 & GST-C3d-sM2 & $8 / 10$ & 20 \\
3 & GST-C3d-L1-SM2 & $9 / 10$ & 10 \\
4 & GST-C3d-L1-C3d-sM2 & $9 / 10$ & 10 \\
5 & GST-C3d-L1-C3d-L2-sM2 & $7 / 10$ & 30 \\
\hline
\end{tabular}

Mice were immunized thrice with GST-sM2, GST-C3d-sM2, GST-C3d-L1-sM2, GST-C3d-L1-C3d-sM2, GST-C3d-L1-C3d-L2-sM2 and GSTSM2+IFA, respectively. Then all the mice were challenged with $10^{6.5} \mathrm{EID}_{50}$ of the A/chicken/Bei Jing/WD9/98 (H9N2) virus. On the indicated days, mice were euthanatized, lung samples were collected and subsequently homogenized and inoculated in SPF chicken embryos for the presence of infectious virus. Virus isolation was operated as described in the Materials and Methods. The mouse was considered to be protected whichever HA test was negative in two virus isolations. The protections from immunized mice on day 5 p.i. are shown.

0.01). The lymphocytes in each group stimulated with Con A showed no significant differences.

\section{Discussion}

Many researchers have confirmed that in the immune system of mammals, complement component C3d as a molecular adjuvant bridging innate and acquired immunity $[7,22,23]$. Apart from enhancing the activation of $B$ cells, promoting the affinity maturity of antibodies and maintaining immunologic memory, C3d also has the molecular adjuvant function of enhancing the antigen presentation of $B$ cells as well as decreasing the activation threshold of these cells [24-26]. As a molecular adjuvant, C3d can be linked to different antigens, effectively enhancing the titer and life-span of specific antibodies against these antigens [7,27-29]. However, there have

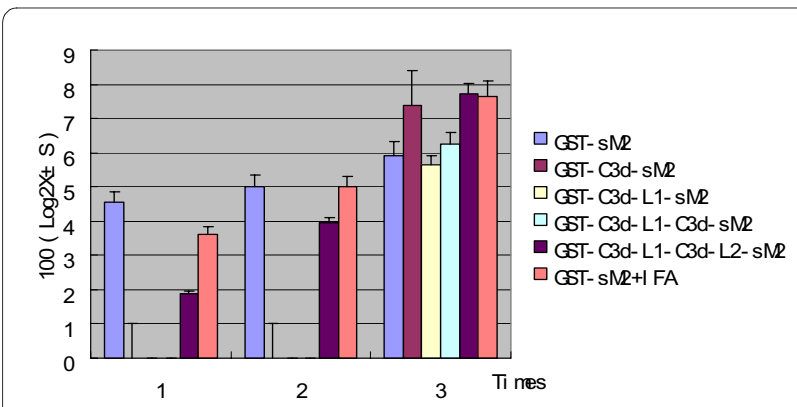

Figure 5 Titers of antibody against $s M 2$ in BALB/c mouse serum detected by ELISA. Sixty BALB/C mice aged 6 weeks were divided into $6(n=10)$ groups. The mice in the six test groups were immunized thrice with GST-SM2, GST-C3d-sM2, GST-C3d-L1-sM2, GST-C3d-L1C3d-sM2, GST-C3d-L1-C3d-L2-sM2 and GST-sM2+IFA, respectively. Blood samples were collected before each vaccination and two weeks after the final booster, and antibody against $\mathrm{SM} 2$ were detected by ELISA. Ab-positive cut-off values were set as mean +2 SD of non-immunized sera. An ELISA Ab titer was expressed as the highest serum dilution giving a positive reaction. The titer values were showed in 100 $(\log 2 X \pm S)$ been few reports about the structure and function of chicken C3d published to date. In 2005, we cloned the full-length chicken $C 3 d$ gene and submitted its sequence to the GenBank database (Accession number: DQ291160). Amino acid homology between chicken C3d and mammal C3d was found to be low.

In this study, we fused one to two copies of $\mathrm{C} 3 \mathrm{~d}$ to the $5^{\prime}$ end of $s M 2$ gene of AIV to construct expression plasmids, from which fusion proteins were expressed and purified. We immunized BALB/c mice and SPF chickens with the purified proteins. The experimental results in mice and chickens showed that C3d could enhance the antibody titer to $\mathrm{sM} 2$ protein in both host species. In studies using human and mouse C3d, most researchers considered that at least three copies of C3d fused to antigen were needed to enhance immunity [30-32]. Suradhat also reported that two copies of mouse C3d fused to bovine rotavirus (BRV) VP7 or bovine herpesvirus type I (BHV-I) glycoprotein D did not enhance antibody titers to either antigen [33]. Bower reported that there was no relationship between the copy numbers of C3d binding to antigen and the antibody titer [34]. In our present study, no obvious changes in antibody levels were detected in each C3d injected group after the first and second immunizations, so repeated immunizations were performed to improve the immune response. However, after the third immunization, both one copy and two copies of C3d significantly enhanced the antibody titer against sM2. This indicates that repeated immunization with chicken C3d can enhance the humoral immune response to an antigen molecule. The antibody titers of sM2 produced by immunizing the fusion proteins of two copies of C3d and sM2 bound by two simple amino acid flexible peptides, are significantly higher than for other treatment groups. This result was further verified by immunization of SPF chickens, and agreed with the findings of a previous report [35]. It is not clear why in mice immunized with fusion 
Table 2: Results of protection from immunized chickens after challenge.

\begin{tabular}{llcc}
\hline Groups & Vaccine & Number of positive/total & Protection ratio (\%) \\
\hline 1 & GST-sM2 & $0 / 15$ & 0 \\
2 & GST-C3d-L1-C3d-L2-sM2 & $3 / 15$ & 20 \\
3 & pcDNA-sM2 & $1 / 15$ & 6.7 \\
4 & pcDNA-C3d-L1-C3d-L2-sM2 & $2 / 15$ & 13.3 \\
5 & GST-SM2+IFA & $4 / 15$ & 26.7 \\
\hline
\end{tabular}

Chickens were immunized thrice with GST-sM2, GST-C3d-L1-C3d-L2-sM2, pcDNA-sM2, pcDNA-C3d-L1-C3d-L2-sM2 and GST-sM2+IFA, respectively. Then all the Chickens were challenged with $5 \times 10^{6.5} \mathrm{EID}_{50}$ of the A/chicken/Bei Jing/WD9/98 (H9N2) virus. On the indicated days, chickens were euthanatized, lung samples were collected and subsequently homogenized and inoculated in SPF chicken embryos for the presence of infectious virus. Virus isolation was operated as described in the Materials and Methods. The chicken was considered to be protected whichever HA test was negative in two virus isolations. The protections from immunized mice on day 5 p.i. are shown.

proteins bearing one copy of C3d or with fusion proteins that consisted of two copies of C3d only linked with one flexible peptide, anti-sM2 antibody could not be detected after two immunizations. It is possible that, because the molecular weight of C3d is greater than that of sM2, the C3d stereochemistry blocked sM2 binding. In contrast, with the protein GST-C3d-L1-C3d-L2-sM2, which has two flexible peptides, repeated immunizations are not required, a finding that has been reported previously [3436].

Many previous researches have shown the molecular adjuvant effect of $\mathrm{C} 3 \mathrm{~d}$ in the immunization of DNA vaccines [33,36-39]. However, our immunological test in SPF chickens found that C3d can enhance the immunogenicity of the linked sM2 protein, whether it exists as a prokaryotic expressed fusion protein or a eukaryotic recombinant plasmid. The reason for this was not clear. We also found that DNA vaccine bearing two copies of C3d can enhance the immunogenicity of the antigen sM2,

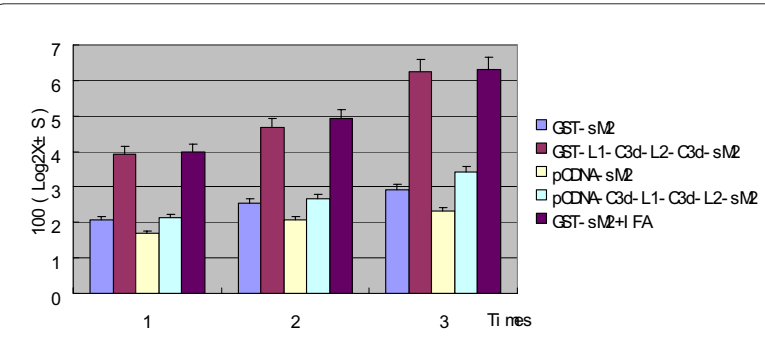

Figure 6 Titers of antibody against SM2 in SPF chicken serum detected by ELISA. Seventy-five SPF chickens aged 3 weeks were divided into $5(n=15)$ groups. The chickens in the five test groups were immunized thrice with GST-sM2, GST-C3d-L1-C3d-L2-sM2, pCDNAsM2, pcDNA-C3d-L1-C3d-L2-sM2 and GST-sM2+IFA, respectively. Blood samples were collected before each vaccination and two weeks after the final booster, and antibody against sM2 were detected by ELISA. Ab-positive cut-off values were set as mean +2 SD of non-immunized sera. An ELISA Ab titer was expressed as the highest serum dilution giving a positive reaction. The titer values were showed in 100 $(\log 2 X \pm S)$ but its ability to increase the antibody titer is lower than that of the fusion proteins.

The transformation ability of lymphocytes may reflect the immune status of the body. Mitsuyoshi reported that C3d might enhance the immunogenicity of antigens without $\mathrm{T}$ lymphocytes [40]. In the present study, we used LPS and Con A as mitogens to perform peripheral blood lymphocyte transformation tests. The results showed that when stimulated with LPS, the differences between the GST-C3d-L1-C3d-L2-sM2 immunized group and the matching control group and between the pcDNA-C3dL1-C3d-L2-sM2 immunized group and the matching control group were significant. However, when stimulated with Con A, there was no significant difference between the immunized groups. Since LPS and Con A are major mitogens of $\mathrm{B}$ and $\mathrm{T}$ lymphocytes respectively, these findings indicate that $\mathrm{C} 3 \mathrm{~d}$ can enhance the proliferation of chicken B lymphocytes but has no obvious effect on the proliferation of $\mathrm{T}$ lymphocytes. In contrast, Mitchel reported that $\mathrm{C} 3 \mathrm{~d}$ could activate $\mathrm{T}$ cells and express major histocompatibility complex class I and II proteins in an antigen dependent manner [29]. Also, Bower reported that $\mathrm{C} 3 \mathrm{~d}$ could induce higher expression of $\mathrm{T}$ cells stimulating secretions of interferon- $\gamma$ and interleukin-4 [41]. Based on this research, our future studies will focus on the effect of chicken complement component C3d on cell-mediated immunity.

In this review, though the results presented here suggest that $\mathrm{C} 3 \mathrm{~d}$ as an adjuvant might be effective to enhance antibody response, a good AIV vaccine is the ability to protect vaccined animals. In order to improve the vaccine efficacy, one or two copies of chicken $C 3 d$ and flexible peptides were applied in the constructs of vaccines, but the M2 vaccines still needs at least two booster immunizations. Furthermore, Vaccinations as described here, only afforded $30 \%$ protection in mice and $20 \%$ protection in chickens. Therefore, this is not a promising vaccine in these constructs. We hope that it is help- 
Table 3: Results of the lymphocyte transformation test.

\begin{tabular}{|c|c|c|}
\hline \multirow[t]{2}{*}{ Groups } & \multicolumn{2}{|c|}{ Mean OD value ${ }^{\mathrm{a}}$} \\
\hline & LPS & Con A \\
\hline pcDNA-sM2 & $0.163 \pm 0.011^{\mathrm{A}}$ & $0.226 \pm 0.015^{\mathrm{A}}$ \\
\hline GST-sM2 & $0.283 \pm 0.009^{A C *}$ & $0.272 \pm 0.013^{\mathrm{A}}$ \\
\hline pcDNA-C3d-L1-C3d-L2-sM2 & $0.533 \pm 0.012^{\mathrm{B} * *}$ & $0.314 \pm 0.011^{\mathrm{A}}$ \\
\hline GST-C3d-L1-C3d-L2-sM2 & $0.962 \pm 0.023^{\mathrm{D} * *}$ & $0.321 \pm 0.011^{\mathrm{A}}$ \\
\hline GST-sM2+IFA & $0.981 \pm 0.023^{E * *}$ & $0.318 \pm 0.011^{\mathrm{A}}$ \\
\hline
\end{tabular}

$A, B, C, D$ and $E$ indicate a significant difference between each group. A value represent means $\pm S D$ of each group $(n=15)$. ${ }^{*}$ Significantly different from the control groups $(p<0.05)$ by ELISA. ${ }^{* *}$ Significantly different from the control groups $(p<0.01)$ by ELISA.

ful to develop a good universal AIV vaccine based on the M2 protein.

\section{Conclusion}

In this study, we investigated the potential of M2-avian C3d fusion proteins to provide effective immunity. These results indicated that chicken $\mathrm{C} 3 \mathrm{~d}$ enhanced the humoral immunity against AIV M2 protein either fused proteins expressed by the prokaryotic system or with the DNA vaccine. Nevertheless, in view of the poor protection ratio for these animals, we speculated that this is not a worthy developing of vaccine in these constructs.

\section{Methods}

Cloning and sequencing of the chicken C3d gene fragment Using the publicly available sequences of human, mouse, hamster, cow, rabbit, pig, chimpanzee and sheep complement component C3d and the fowl complement component C3, two primers (upstream: 5'-CCT GGT GGA GAA AGC C-3'; downstream: 5'-TGC GGT AGG TGA TGG C-3') were designed. Samples of liver tissue were obtained from a specific-pathogen-free (SPF) White Leghorn chicken inoculated with a $0.5 \mathrm{ml}$ dose of infectious coryza oil vaccine. RNA was extracted using Trizol reagent (Invitrogen, USA) as per the manufacturer's instructions. A reverse transcriptase (RT) reaction was carried out using the above reverse primer and Maloney murine leukemia virus reverse transcriptase (Invitrogen, USA) to generate cDNA containing the chicken complement C3d sequence. The C3d gene was PCR amplified using the above cDNA as a template. Standard PCR conditions consisted of an initial denaturation step at $94^{\circ} \mathrm{C}(5$ $\mathrm{min})$, followed by 30 cycles of denaturing at $94^{\circ} \mathrm{C}(30 \mathrm{sec})$, annealing at $56^{\circ} \mathrm{C}(45 \mathrm{sec})$ and extending at $72^{\circ} \mathrm{C}(1 \mathrm{~min})$, followed by a $10 \mathrm{~min}$ final extension step at $72^{\circ} \mathrm{C}$. The plasmid sequence was verified by automated nucleotide sequence analysis using standard protocols. The RT-PCR product was inserted into pMD18-T (Takara) by TA- cloning to facilitate subsequent manipulation, and the recombined plasmid was designated pMD-cC3d.

\section{Construction and identification of the recombinant expression plasmids}

Primers were designed for PCR amplification of the fulllength gene sequence of (918 bp) chicken C3d (cC3d) from the sequence of the recombinant plasmid pMDcC3d. The forward primer (5'-GAATTC ATGCACCTCATTGTGACCCCCTCGGGCAGT-3') was specific for the 5 ' coding region of the chicken $C 3 d$ gene and began with an upstream EcoRI restriction site (in bold) and start code. The reverse primer (5'-CCCGGGGCGGTAGGTGATGGCGTTG-3') coded for the 3' region and provided an $X m a$ I restriction site (in bold). The $s M 2$ gene of AIV (A/chicken/Guangdong/2000, H9N2) was amplified from plasmid pMD-sM2 by PCR [42]. The amplified $s M 2$ gene fragment (256 bp in length) encoded the complete M2 open reading frame of AIV but not the transmembrane domain. Upstream HindIII and downstream XhoI sites were added to the sM2 fragment. Two linker sequences (L1 and L2) were constructed following the design of Dempsey et al. [7] to provide spacing between protein motifs. Four single stranded oligonucleotides encoding the amino acid sequence $\mathrm{GS}\left[\mathrm{G}_{4} \mathrm{~S}\right]_{2} \mathrm{GS}$ with upstream $X m a \mathrm{I}$ and downstream HindIII or NheI sites were prepared by forming adhesive ends after annealing. One or two copies of the $c C 3 d$ and $s M 2$ genes were joined with different linkers to form four gene expression cassettes: EcoRI-C3d-HindIII-sM2-XhoI, EcoRI-C3d-XmaI-L2HindIII-sM2-XhoI, EcoRI-C3d-XmaI-L1-NheI-C3d-HindIII-sM2-XhoI and EcoRI-C3d-XmaI-L1-NheI-C3dXmaI-L2-HindIII-sM2-XhoI. To construct the plasmids needed, these gene expression cassettes were ligated into EcoRI and XhoI sites of a plasmid vector pGEX-5x-1 (Amersham-Pharmacia). The gene cassette encoding EcoRI-C3d-XmaI-L1-NheI-C3d-XmaI-L2-HindIII-sM2-

$X h o I$ was cloned into the identical site of the eukaryotic 
plasmid pcDNA4.0-Lacz (Invitrogen) containing the cytomegalovirus immediate-early promoter, an upstream intron and the simian virus 40 late poly (A) signal sequences. As a control, the $s M 2$ gene alone was inserted into plasmids pGEX-5x-1 and pcDNA4.0-Lacz respectively to construct recombinant expression plasmids $[43,44]$.

\section{Expression and purification of fusion proteins}

The recombinant prokaryotic expression plasmids were transformed into competent Escherichia coli strain BL21 (DE3). Single colonies of four positive recombinant bacteria were inoculated into Luria broth (LB) supplemented with $100 \mu \mathrm{g} / \mathrm{ml}$ ampicillin. These cultures were incubated at $37^{\circ} \mathrm{C}$ until mid-log phase $\left(\mathrm{OD}_{600} 0.6-0.8\right)$. The broth was then divided into two, with one half being induced with $1 \mathrm{mM}$ isopropyl $\beta$-D-thiogalactoside (IPTG) while the other half served as an uninduced control. The broths were incubated for an additional $4 \mathrm{~h}$ at $28^{\circ} \mathrm{C}$. The expression product was purified from the supernatants of bacterial lysates by affinity chromatography using glutathione sepharose 4B (Amersham-Pharmacia). Protein concentrations were determined with a Bio-Rad protein assay dye reagent concentrate, and the purified proteins were stored at $-80^{\circ} \mathrm{C}$.

\section{SDS-PAGE and Western immunoblot detection of fusion proteins}

Expressed proteins were separated by electrophoresis in $12 \%$ sodium dodecyl sulfate (SDS)-polyacrylamide gels. To visualize total proteins, gels were stained with Bio$\mathrm{Safe}^{\mathrm{TM}}$ Coomassie G250 stain (New England Biolabs). Protein All Blue ${ }^{\mathrm{rm}}$ standard (New England Biolabs) was used as a molecular mass standard.

For Western immunoblot analysis, proteins were transferred to nitrocellulose membranes (Millipore). The membranes were blocked for a minimum of $1 \mathrm{~h}$ with PBS containing $0.5 \%$ bovine serum albumin (BSA) (Sigma) and $0.1 \%$ Tween 20 (Sigma) prior to incubation with a 1:500 dilution of a monoclonal antibody (3F8) specific for sM2 (primary antibody, which is prepared in previous research by ourselves). After extensive washing, bound antibodies were detected by enhanced chemiluminescence (Amersham) with a 1:5000 dilution of horseradish peroxidase-conjugated anti-mouse IgG (Sigma) (secondary antibody). Blots were developed using alkaline phosphatase (AP) Conjugate Substrate Kit (New England Biolabs). Color development was stopped by washing the membrane in deionized water.

\section{Expression of recombinant eukaryotic plasmids in BHK-21 cells}

The eukaryotic plasmids containing EcoRI-C3d-XmaIL1-NheI-C3d-XmaI-L2-HindIII-sM2-XhoI and the sM2 gene alone were isolated from $E$. coli DH5á cells and puri- fied using a Plasmid Mini Purification Kit (QIAGEN $\mathrm{GmbH})$. The endotoxin-free plasmids were verified by restriction endonuclease digestion with EcoRI and XhoI and analysis by gel electrophoresis. The purity and concentration of DNA preparations was determined based on the optical densities (ODs) at 260 and $280 \mathrm{~nm}$. In a sixwell plate (Costar), baby hamster kidney cell lines (BHK21) on slides were transfected with $4.0 \mu \mathrm{g}$ of DNA and 10 $\mu \mathrm{l}$ of Lipofectamine 2000 (Life Technologies, Grand Island, N. Y.) according to the manufacturer's guidelines. The transfected cells were incubated for $36 \mathrm{~h}$ at $37^{\circ} \mathrm{C}$ in a $\mathrm{CO}_{2}$ incubator, and then harvested for immunological examination.

\section{Indirect immunofluorescence detection}

The slides that carried the infected cells were carefully removed from the wells, washed with $0.01 \mathrm{M} \mathrm{PBS}$, then fixed (acetone: absolute alcohol, 3:2) for $30 \mathrm{~min}$ at $4^{\circ} \mathrm{C}$. The fixed BHK-21 cells were incubated with a 1:100 dilution of monoclonal antibody (3F8) against sM2 for 45 min at $37^{\circ} \mathrm{C}$ in a wet box. After extensive washing, bound antibodies were detected using a FITC-conjugated goat anti-mouse IgG (Sigma) and observed by fluorescence microscopy.

\section{Animals immunization and challenge}

Six-week-old BALB/c mice (Jingfeng Medical Laboratory Institute, Beijing, China) were inoculated. Mice were randomly divided into six groups, with 10 mice in each group, and housed with free access to food and water. Mice were administered with purified proteins GST-sM2, GST-C3d-sM2, GST-C3d-L1-sM2, GST-C3d-L1-C3dsM2 and GST-C3d-L1-C3d-L2-sM2 in $100 \mu$ of PBS subcutaneously in the rear flank. Mice were also injected with GST-sM2 in incomplete Freund's adjuvant (IFA; Sigma) as control group. Booster immunizations with the same proteins were performed with an interval of two weeks. For the three inoculations, the injection dose was $100 \mu \mathrm{g}$ proteins respectively. Non-lethal tail bleeds were collected before each vaccination and two weeks after the final booster. For virus challenge, phenobarbital sodiumanesthetized mice were intranasally infected with A/ chicken/Bei Jing/WD9/98 (H9N2) virus $\left(10^{6.5}\right.$ EID $_{50}$ [the $50 \%$ embryo infectious dose]) in $100 \mu$ l of PBS per mouse 2 weeks after the final immunization. Lung samples were collected from individual mice at day 5 after a challenge infection. The whole-lung extracts prepared as homogenates using frosted glass slides were centrifuged at 3,000 rpm for 5 min to collect supernatants. The lung supernatants were frozen and kept at $-70^{\circ} \mathrm{C}$ until used for virus isolation assay. The supernatants $(0.2 \mathrm{ml} / \mathrm{egg})$ were inoculated into the allantoic cavity of five 10-days old specific pathogen free (SPF) chicken embryos. Early embryonic death within the first 24 hours of inoculation was consid- 
ered as non-specific death and these embryos were discarded. After incubation at $37^{\circ} \mathrm{C}$ for 5 days the allantoic fluid was harvested and tested by haemagglutination (HA) assay. In the cases there was no virus was detected in the first virus isolation, the allantoic fluid was passaged once in embryonated hen eggs. The mouse was considered not to be protected whichever HA test was positive in two virus isolations. The mouse was considered to be protected when the second passage HA test still was negative.

75 aged 3 weeks SPF chickens (Beijing Center for Laboratory Animals, Beijing) were divided into five equal groups. Three groups of chickens were injected intramuscularly with $200 \mu \mathrm{g}$ of purified proteins GST-sM2, GSTsM2 mixed with incomplete Freund's adjuvant (IFA; Sigma) and GST-C3d-L1-C3d-L2-sM2 respectively. The other two groups were vaccinated with the mixtures of the recombinant plasmids DNA (pcDNA-sM2 and pcDNA-C3d-L1-C3d-L2-sM2) and Lipofectamine ${ }^{\mathrm{mm}} 2000$ (Invitrogen) by the intramuscular route. A dose of $0.2 \mathrm{ml}$ contained $100 \mu \mathrm{g}$ DNA and $50 \mu \mathrm{l}$ Lipofectamine $^{\mathrm{mm}} 2000$ in Opti-MEM ${ }^{\circ}$ I Reduced Serum Medium (Invitrogen). Two booster immunizations of the same purified proteins and plasmid DNA were given at an interval of two weeks, with the same dose being $100 \mu \mathrm{g}$ respectively. Blood was collected from the wing vein before every vaccination and two weeks after the final boost. Virus challenge and virus isolation trials were carried out refer to the above mouse test procedure, $5 \times 10^{6.5} \mathrm{EID}_{50}$ of $\mathrm{H} 9 \mathrm{~N} 2$ virus was used as the challenge viral dosage. All of the animal experiments performed in this study were approved by the Beijing Laboratory Animal Management Office (China).

\section{Indirect ELISA for detection of anti-sM2 antibodies}

According to previously described methods [15,43], purified sM2 protein was obtained by cleaving GST-sM2 bound to Glutathione Sepharose 4B (Amersham-Pharmacia) with factor Xa. Polystyrene microplates (Costar, Corning Incorporated) were coated with $100 \mu \mathrm{l}$ of purified sM2 protein at a concentration of $0.125 \mu \mathrm{g} / \mathrm{ml}$, and the samples were incubated with 2 -fold serially diluted sera from the vaccinated animals. Anti-mouse (or antichicken) IgG conjugated to horseradish peroxidase (Sigma, 1:8000 dilutions) was used as the secondary antibody. End-point titers were calculated as the reciprocal of the last serum dilution that gave a value 2 -fold higher than the negative serum. Antibody titers below the cutoff of the assay were assigned an arbitrary titer one-half the cutoff in order to allow calculation of the geometric mean of the titers.

\section{Lymphocyte proliferation assay}

Ten SPF chickens from each of the immunized groups described above were bled aseptically from the heart two weeks after the final boost. Peripheral blood lymphocytes were isolated by Lympholyte ${ }^{\circ}$-Mammal (Cedarlane, Laboratories Limited). The cells were resuspended in RPMI 1640 medium supplemented with $10 \%$ fetal calf serum, $100 \mu \mathrm{g} / \mathrm{ml}$ penicillin and $100 \mu \mathrm{g} / \mathrm{ml}$ streptomycin (all from Invitrogen). Freshly isolated lymphocytes were seeded in quadruplicate at $4 \times 10^{6} \mathrm{cells} / \mathrm{ml}$ in 96-well round-bottomed microtiter plates (Costar). Cells were either left un-stimulated or stimulated with lipopolysaccharide (LPS; $5 \mu \mathrm{g} / \mathrm{ml}$; Sigma) or Concanavalin A (Con A; $5 \mu \mathrm{g} / \mathrm{ml}$; Sigma) as the mitogen. After incubation for $60 \mathrm{~h}$ at $37^{\circ} \mathrm{C}$, 3- [4, 5-dimethylthiozol-2-yl]-2, 5-diphenylthtrazolium bromide (MTT) was added to the cultures and a further $3 \mathrm{~h}$ incubation performed. The reaction was

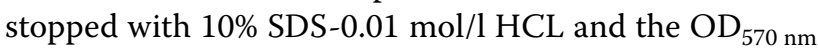
was measured using a Bio-Rad 550 plate reader.

\section{Statistical analysis}

The anti-sM2 antibody titers were expressed as the 100 $\log _{2} X$ where $\mathrm{X}$ was the reciprocal of the final dilution of serum. Proliferation of lymphocytes were expressed as the $\mathrm{OD}_{570}$ value which was expressed as the mean \pm standard errors of the mean (SEM) of at least three independent experiments. The $t$-test was used to analyze the statistical significance of the differences. Probability $(P)$ values of $<0.05\left(^{*}\right)$ and $<0.01\left(^{* * *}\right)$ were considered significant.

\section{List of abbreviations used}

sM2: M2 protein with the transmembrane region deleted; GST: Glutathione S-transferase tagged proteins in pGEX expression vector; ELISA: enzyme-linked immunosorbent assay; SPF: specific pathogen free; CR2: cell receptor 2; HIV-1: human immunodeficiency virus type 1; IPTG: isopropyl $\beta$-D-thiogalactoside; BSA: bovine serum albu$\min$.

\section{Competing interests}

The authors declare that they have no competing interests.

\section{Authors' contributions}

ZHZ carried out most of the experiments and wrote the manuscript. YQL and FYC carried out study design, also wrote and revised the manuscript. SFX and LZ helped in experiments, participated in antibody detection and statistical analyses. BYJ and XLC conceived the study, and participated in its design and coordination, also helped to look over the manuscript. All authors read and approved the final manuscript.

\section{Acknowledgements}

This work was supported by the National Natural Science Foundation of China (Grant numbers: 30440011 and 30671560) and the Natural Science Foundation of Beijing (Grant number: 5082006). A special word of thanks to Patrick Blackall from the Department of Primary Industries and Fisheries Animal Research Institute (Queensland, Australia) who carefully read the manuscript and gave us very useful comments. 


\section{Author Details}

${ }^{1}$ College of Animal Medicine, China Agricultural University, Beijing 100094, China and 2 Institute of Animal Husbandry and Veterinary Medicine, Beijing Academy of Agricultural and Forestry Sciences, Beijing 100097, China

Received: 18 March 2010 Accepted: 9 May 2010

Published: 9 May 2010

\section{References}

1. Nielsen $\mathrm{CH}$, Fischer EM, Leslie RG: The role of complement in the acquired immune response. J Immunol 2000, 100:4-12.

2. Rus $\mathrm{H}$, Cudrici $\mathrm{C}$, Niculescu F: The role of the complement system in innate immunity. Immunol Res 2005, 33:103-12.

3. Thornton BP, Vetvicka V, Ross GD: Natural antibody and complementmediated antigen processing and presentation by B lymphocytes. J Immunol 1994, 152:1727-1737.

4. Sahu A, Sunyer JO, Moore WT, Sarrias MR, Soulika AM, Lambris JD: Structure, functions, and evolution of the third complement component and viral molecular mimicry. Immunol Res 1998, 17:109-121

5. Sun-HL Jae UJ, Robert EM: Complementing viral infection: mechanisms for evading innate immunity. Trends in Microbiology 2003, 11:449-452.

6. Thornton BP, Vetvicka V, Ross GD: Function of C3 in a humoral response: $\mathrm{iC} 3 \mathrm{~b} / \mathrm{C} 3 \mathrm{dg}$ bound to an immune complex generated with natural antibody and a primary antigen promotes antigen uptake and the expression of co-stimulatory molecules by all B cells, but only stimulates immunoglobulin synthesis by antigen-specific B cells. Clin Exp Immunol 1996, 104:531-537.

7. Dempsey PW, Allison ME, Akkaraju S, Goodnow CC, Fearon DT: C3d of complement as a molecular adjuvant: bridging innate and acquired immunity. Science 1996, 271:348-350.

8. Lou D, Kohler H: Enhanced molecular mimicry of CEA using photoaffinity cross-linked C3d peptide. Nature Biotechnology 1998 , 16:458-462

9. Ross $T M, X u Y$, Bright RA, Robinson HL: C3d enhancement of antibodies to hemagglutinin accelerates protection against influenza virus challenge. Nature Immunol 2000, 1:127-131.

10. Anderson R, Gao XM, Papakonstantinopoulou A, Fairweather N, Roberts $M$, Dougan G: Immunization of mice with DNA encoding fragment $C$ of tetanus toxin. Vaccine 1997, 15:827-829.

11. Bower J F, Sanders K L, Ross T M: C3d Enhances Immune Responses Using Low Doses of DNA Expressing the HIV-1 Envelope from CodonOptimized Gene Sequences. Curr HIV Res 2005, 3:191-198.

12. Ross TM, Xu Y, Green TD, Montefiori DC, Robinson HL: Enhanced Avidity Maturation of Antibody to Human Immunodeficiency Virus Envelope: DNA Vaccination with gp120-C3d Fusion Proteins. AIDS Res Hum Retroviruses 2001, 617:829-835.

13. Wang L, Oriol Sunyer J, Bello LJ: Immunogenicity of a bovine viral diarrhea virus E2-C3d fusion protein containing a bovine homolog of C3d. Developmental and comparative immunology 2005, 29:907-915.

14. Liu D, Niu ZX: Cloning of a gene fragment encoding chicken complement component C $3 \mathrm{~d}$ with expression and immunogenicity of Newcastle disease virus F gene-C3d fusion protein. Avian Pathol 2008, 37:477-485

15. Logan GJ, Wang L, Zheng M, Coppel RL, Alexander IE: Antigen fusion with C3d3 augments or inhibits humoral immunity to AAV genetic vaccines in a transgene-dependent manner. Immunol Cell Biol 2010, 88:228-32.

16. Slepushkin VA, Katz JM, Black RA, Gamble WC, Rota PA, Cox NJ: Protection of mice against influenza $A$ virus challenge by vaccination with baculovirus expressed M2 protein. Vaccine 1995, 13:1399-1402.

17. Frace AM, Klimov Al, Rowe T, Black RA, Katz JM: Modified M2 proteins produce heterotypic immunity against influenza A virus. Vaccine 1999, 17:2237-2244

18. Neirynck S, Deroo T, Saelens X, Vanlandschoot P, Jou WM, Fiers W: A universal influenza $A$ vaccine based on the extracellular domain of the M2 protein. Nat Med 1999, 5:1157-1163.

19. Zhao G, Lin Y, Du L, Guan J, Sun S, Sui H, Kou Z, Chan CC, Guo Y, Jiang S, Zheng BJ, Zhou Y: An M2e-based multiple antigenic peptide vaccine protects mice from lethal challenge with divergent $\mathrm{H} 5 \mathrm{~N} 1$ influenza viruses. Virol J 2010, 18:7-9.

20. Rao SS, Kong WP, Wei CJ, Van Hoeven N, Gorres JP, Nason M, Andersen H, Tumpey TM, Nabel GJ: Comparative efficacy of hemagglutinin, nucleoprotein, and matrix 2 protein gene-based vaccination against H5N1 influenza in mouse and ferret. PLoS One 2010, 5:e9812.

21. Mozdzanowska K, Feng J, Eid M, Kragol G, Cudic M, Otvos L Jr, Gerhard W: Induction of influenza type $A$ virus-specific resistance by immunization of mice with a synthetic multiple antigenic peptide vaccine that contains ectodomains of matrix protein 2. Vaccine 2003, 21:2616-2626.

22. Nauta AJ, Roos A, Daha MR: A regulatory role for complement in innate immunity and autoimmunity. Int Arch Allergy Immunol 2004 134:310-323.

23. Haas KM, Toapanta FR, Oliver JA, Poe JC, Weis JH, Karp DR, Bower JF, Ross TM, Tedder TF: Cutting edge: C3d function as a molecular adjuvant in absence of CD21/35 expression. J Immunol 2004, 172:5833-5839.

24. Carter $\mathrm{RH}$, Fearon DT: CD19: lowering the threshold for antigen receptor stimulation of B lymphocytes. Science 1992, 256:105-107.

25. Hess MW, Schwendinger MG, Eskelinen EL, Pfaller K, Pavelka M, Dierich MP, Prodinger WM: Tracing uptake of $\mathrm{C} 3 \mathrm{dg}$-conjugated antigen in $\mathrm{B}$ cells via complement receptor type 2(CR2, CD21). Blood 2000, 95:2617-2623.

26. Cherukuri A, Cheng PC, Pierce SK: The role of the CD19/CD21 complex in B cell processing and presentation of complement: Tagged antigen. J Immunol 2001, 167:163-172

27. Lee $Y$, Haas KM, Gor DO, Ding X, Karp DR, Greenspan NS, Poe JC, Tedder TF: Complement Component C3d-Antigen Complexes Can Either Augment or Inhibit B Lymphocyte Activation and Humoral Immunity in Mice Depending on the Degree of CD21/CD19 Complex Engagement. J Immunol 2005, 75:8011-8023.

28. Green TD, Montefiori DC, Ross TM: Enhancement of antibodies to the human immunodeficiency virus type 1 envelope by using the molecular adjuvant C3d. J Virol 2003, 77:2046-2055.

29. Mitchell JA, Green TD, Bright RA, Ross TM: Induction of heterosubtypic immunity to influenza A virus using a DNA vaccine expressing hemagglutinin-C3d fusion proteins. Vaccine 2003, 21:902-914

30. Green TD, Newton BR, Rota PA, Xu Y, Robinson HL, Ross TM: C3d enhancement of neutralizing antibodies to measles hemagglutinin. Vaccine 2002, 20:242-248.

31. Henson SE, Smith D, Boackle SA, Holers VM, Karp DR: Generation of recombinant human $\mathrm{C} 3 \mathrm{dg}$ tetramers for the analysis of $\mathrm{CD} 21$ binding and function. J Immunol Methods 2001, 258:97-107.

32. Frinklln R, Ross TM: Mouse strain-dependent differences in enhancement of immune response by C3d. Vaccine 2004 22:1773-1778

33. Suradhat S, Braun RP, Lewis PJ, Babiuk LA, Hurk S van Drunen Littel-van den, Griebel PJ, Baca-Estrada ME: Fusion of C3d molecule with bovine rotavirus VP7 or bovine herpesvirus type 1glycoprotein D inhibits immune responses following DNA immunization. J Vet Immunol 2001, 83:79-92.

34. Bower JF, Yang X, Sodroski J, Ross TM: Elicitation of neutralizing antibodies with DNA vaccines expressing soluble stabilized human immunodeficiency virus type 1 envelope glycoprotein trimers conjugated C3d. J virol 2004, 78:4710-4719.

35. Green TD, Montefiori DC, Ross TM: Enhancement of Antibodies to the Human Immunodeficiency Virus Type 1 Envelope by Using the Molecular Adjuvant C3d. J Virol 2003, 77:2046-2055.

36. Roberts T, Snow EC: Cutting edge: recruitment of the CD19/CD21 coreceptor to $\mathrm{B}$ cell antigen receptor is required for antigen-mediated expression of $\mathrm{Bcl}-2$ by resting and cycling hen egg lysozyme transgenic B cells. J Immunol 1999, 162:4377-4380.

37. Lambris JD, Ganu VS, Hirani S, Müller-Eberhard HJ: Mapping of the receptor (CR2) binding site and antigenic site in the C3d domain of the third component of complement. Proc Natl Acad Sci 1985, 82:4235-4239.

38. Hellwage J, Jokiranta TS, Friese MA, Wolk TU, Kampen E, Zipfel PF, Meri S: Complement $\mathrm{C} 3 \mathrm{~b} / \mathrm{C} 3 \mathrm{~d}$ and cell surface polyanions are recognized by overlapping binding sites on the most carboxyl-terminal domain of complement factor H. J Immunol 2002, 169:6935-6942.

39. Liu F, Mboudjeka I, Shen S, Chou TH, Wang S, Ross TM, Lu S: Independent but not synergistic enhancement to the immunogenicity of DNA vaccine expressing HIV-1 gpl20 glycoprotein by codon optimization and C3d fusion in a mouse mode1. Vaccine 2004, 22:1764-1772.

40. Mitsuyoshi JK, Hu Y, Test ST: Role of complement receptor type 2 and endogenous complement in the humoral immune response to conjugates of complement C3d and pneumococcal serotype 14 capsular polysaccharide. Infect Immun 2005, 73:7311-7316. 
41. Bower JF, Sanders KL, Ross TM: C3d enhances immune responses using low doses of DNA expressing the HIV-1 envelope from codonoptimized gene sequences. Curr HIV Res 2005, 3:191-198.

42. Zhang ZH, Zhou XM, Chen NH, Chen XL, Luo CB, Li YQ: Cloning and Structural Analysis of Chicken Complement Component C3d Gene. Biotechnology Letter 2006, 17:267-270. [In Chinese].

43. Li YQ, Yao SX, Wei L, Yang HC: Deletion of coding sequence of transmembrane domain of M2 protein of Avian Influenza virus. Chinese Journal of Veterinary Medicine 2004, 40:6-9. [In Chinese].

44. Li YQ, Yang J, Wei L, Yang HC: Expression of Avian Influenza Virus M2 Gene in E. coli and Analysis of its antigenicity. Acta Veterinaria et Zootechnica Sinica 2005, 36:739-743. [In Chinese].

doi: $10.1186 / 1743-422 X-7-89$

Cite this article as: Zhang et al., Fusion to chicken C3d enhances the immunogenicity of the M2 protein of avian influenza virus Virology Journal 2010, $7 \cdot 89$

Submit your next manuscript to BioMed Central and take full advantage of:

- Convenient online submission

- Thorough peer review

- No space constraints or color figure charges

- Immediate publication on acceptance

- Inclusion in PubMed, CAS, Scopus and Google Scholar

- Research which is freely available for redistribution

Submit your manuscript at www.biomedcentral.com/submit 